\title{
A UNIFIED MODEL FOR THE SPECTRAL VARIABILITY IN GRS 1915+105
}

\author{
T. Belloni, ${ }^{1}$ M. Méndez, ${ }^{1,2}$ A. R. King, ${ }^{3}$ M. VAn der Klis, ${ }^{1}$ and J. VAn Paradiss ${ }^{1,4}$ \\ Received 1997 July 16; accepted 1997 August 12; published 1997 September 16
}

\begin{abstract}
We have analyzed the spectral variations of the superluminal black hole X-ray binary GRS $1915+105$ by using data obtained with the PCA on the Rossi XTE. We find that, despite the marked differences in the structure and the timescale of variability, all spectral changes can be attributed to the rapid disappearing of the inner region of an accretion disk, followed by a slower refilling of the emptied region. The timescale for each event is determined by the extent of the missing part of the disk. The observed relation between the duration of an event and the radius of the disappearing region matches remarkably well the expected radius dependence of the viscous timescale for the radiation-pressure-dominated region of an accretion disk.
\end{abstract}

Subject headings: accretion, accretion disks — binaries: close — black hole physics — instabilities stars: individual (GRS 1915+105) - X-rays: stars

\section{INTRODUCTION}

The X-ray source GRS $1915+105$ is the best of the two only examples of Galactic objects that show superluminal expansion in radio observations (Mirabel \& Rodríguez 1994). It is located at a distance of $12.5 \mathrm{kpc}$, and its relativistic jets are directed at $70^{\circ}$ from the line of sight (Mirabel \& Rodríguez 1994). After its discovery as an X-ray transient in 1992 (CastroTirado, Brandt, \& Lund 1992), a number of outbursts have been reported, although it is possible that the source has never completely switched off in between. The Rossi X-Ray Timing Explorer (RXTE) started observing GRS 1915+105 in a bright and variable state in 1996 April and continued to observe it regularly at least once per week since then. During this period, the source has displayed a remarkable richness in variability, ranging from quasi-periodic burstlike events, deep regular dips, and strong quasi-periodic oscillations (QPOs), alternated with quiescent periods (Greiner, Morgan, \& Remillard 1996; Morgan, Remillard, \& Greiner 1997; Chen, Swank, \& Taam 1997; Belloni et al. 1997; Taam, Chen, \& Swank 1997). Because of its similarities to the other Galactic superluminal source, GRO 1655-40 (Zhang et al. 1994), whose dynamical mass estimate implies the presence of a black hole (Bailyn et al. 1995), and because of its high luminosity, which exceeds the Eddington limit for a neutron star, the source is suspected to host a black hole.

In a previous paper (Belloni et al. 1997, hereafter Paper I) we showed that the complicated light curve of GRS 1915+105 can be described by the rapid $(\sim 1 \mathrm{~s})$ appearance and disappearance of emission from an optically thick inner accretion disk. In this Letter we report on the results of the analysis of a later observation that allowed a more detailed investigation of the spectral variability of the source. We find that the variation timescale, $t_{\text {rec}}$, of the disk source is completely specified

\footnotetext{
${ }^{1}$ Astronomical Institute "Anton Pannekoek," University of Amsterdam and Center for High-Energy Astrophysics, Kruislaan 403, NL-1098 SJ Amsterdam, the Netherlands.

${ }^{2}$ Facultad de Ciencias Astronómicas y Geofísicas, Universidad Nacional de La Plata, Paseo del Bosque S/N, 1900 La Plata, Argentina.

${ }^{3}$ Astronomy Group, University of Leicester, Leicester LE1 7RH, England, UK.

${ }^{4}$ Department of Physics, University of Alabama in Huntsville, Huntsville, AL 35899.
}

by the maximum size, $R_{\max }$, of its inner radius. The relation between these quantities is precisely as expected for the viscous evolution of a radiation-pressure-dominated accretion disk.

\section{X-RAY OBSERVATIONS}

Among the many PCA observations of GRS 1915+105, that of 1997 June 18 deserves particular attention, since it reproduces within 1 day most of the timing behaviors observed from the source. The data consist of two segments of roughly $1 \mathrm{hr}$ each. Since the two segments are essentially similar, we will restrict ourselves to the second one, which starts at 14:36 UT and ends at 15:35 UT. A $1200 \mathrm{~s}$ representative stretch of the 2.0-40.0 keV light curve, binned to $1 \mathrm{~s}$, can be seen in the top panel of Figure 1. It consists of a sequence of "bursts" of different duration with quiescent intervals in between. All bursts start with a well-defined sharp peak and decay faster than they rise. The longer bursts show oscillation (or subbursts) toward the end. In the following, we define an event as a full cycle of one quiescent interval followed by one burst. With this definition there is no interval between events. In order to maintain precision, separate events are considered to be only those whose quiescent count rate goes below 10,000 counts $\mathrm{s}^{-1}$ in the total $2.0-40.0 \mathrm{keV}$ band. We measured the start of an event (corresponding to the end of the previous one) as the time of the small dip at the end of the decay (see Fig. 1). Since all the bursts start with a sharp peak, the time of the peak can be taken as the separation between the quiescent phase and the burst. Finally, the bottom level of each quiescent part has been measured as the lowest level, excluding the small dip mentioned above. With these numbers it is possible to associate four quantities with an event: total length, length of the quiescent part, length of the outburst, and level of the bottom. Two strong correlations emerge: $(i)$ the longer the event, the lower the bottom level (see below), and (ii) a one-to-one relation exists between quiescent phase and burst duration (Fig. 2). No correlation is seen between a burst duration and the following quiescent interval.

In order to follow the spectral evolution, we produced two X-ray colors: $\mathrm{HR} 1=B / A$ and $\mathrm{HR} 2=C /(A+B)$, where $A, B$, and $C$ are the counts in the $2.0-5.0 \mathrm{keV}, 5.0-13.0 \mathrm{keV}$, and $13.0-40.0 \mathrm{keV}$ intervals, respectively. We produced color-color (C-C) diagrams by selecting events according to their total 

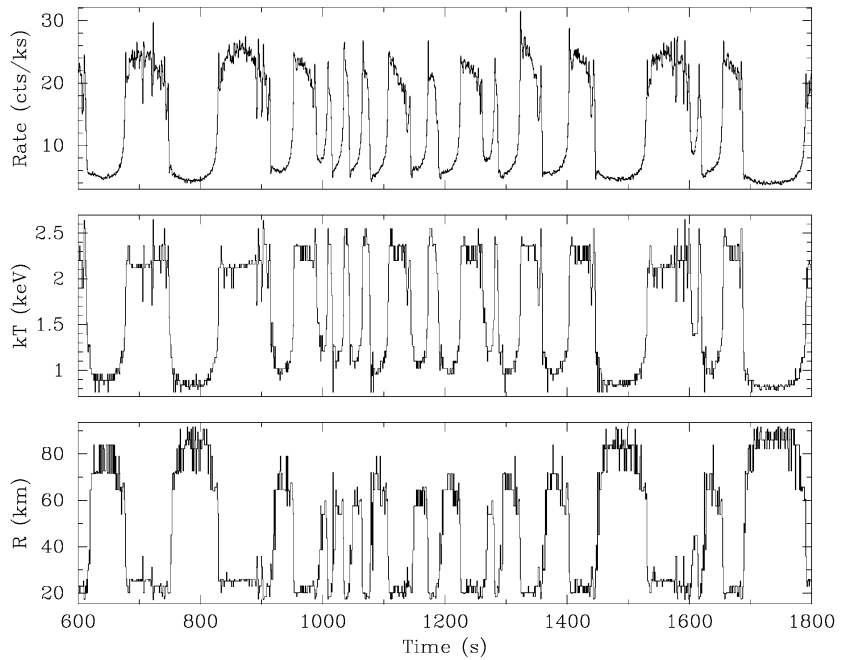

FIG. 1.-Upper panel: $2.0-40.0 \mathrm{keV}$ PCA light curve. Time zero corresponds to 1997 June 18, 14:36 UT. Middle and lower panels: Corresponding inner radius and temperature (see text).

length. It turns out that there is a very strong correlation between the pattern traced out in the C-C diagram by each event and its duration. In Figure 3, one can see two extreme cases and an intermediate one. All other events follow a similar pattern. During short events (top panel) the quiescent period is at the bottom left in the C-C diagram, then the source moves up to the top left during peak, then down and right to the dip. In longer events the sequence is the same, with the only difference being that the quiescent part is located farther to the right the longer the event is. The small oscillations at the end of long bursts show up as oscillations around the peak points in the C-C diagram (Fig. 3, middle and bottom panels).

The spectral evolution is extremely reproducible throughout the different events. We therefore divided the $\mathrm{C}$-C diagram into regions 0.01 wide in HR2 and 0.1 wide in HR1 and accumulated energy spectra for all the (74) populated regions of the diagram. The spectra were accumulated in 48 energy bins with $1 \mathrm{~s}$ time resolution. The background subtraction was performed with a background spectrum extracted from a blank sky observation and normalized to the highest channels where the source signal is absent. We used the latest detector response matrix available (1997 April, Version 2.1.2). A systematic error of $2 \%$ has been added to the data (see Paper I). In order to avoid possible problems due to the background subtraction, we limited our fits to energies below $30.0 \mathrm{keV}$. Following Paper I, for each of the regions we fitted the data with a "standard" spectral model for black hole candidates, consisting of a diskblackbody model (Mitsuda et al. 1984) plus a power law. A broad Gaussian line was added at a fixed energy of $6.3 \mathrm{keV}$ as in Paper I. We found the interstellar absorption to be stable around $4.5 \times 10^{22} \mathrm{~cm}^{-2}$, so it was fixed to that value. Notice that this is lower than the value found in Paper I because of the improved calibration. It is slightly lower than the best-fit ROSAT value (Greiner et al. 1996). The resulting parameters for the power-law component depend on the inclusion of the Gaussian line mentioned above, but the disk-blackbody parameters are independent of this. In the following we will restrict ourselves to the discussion of the latter. Since both distance and inclination of the system are known (the latter is assumed to be equal to the observed inclination of the radio jet; see Mirabel \& Rodríguez 1994), it is possible to derive the inner

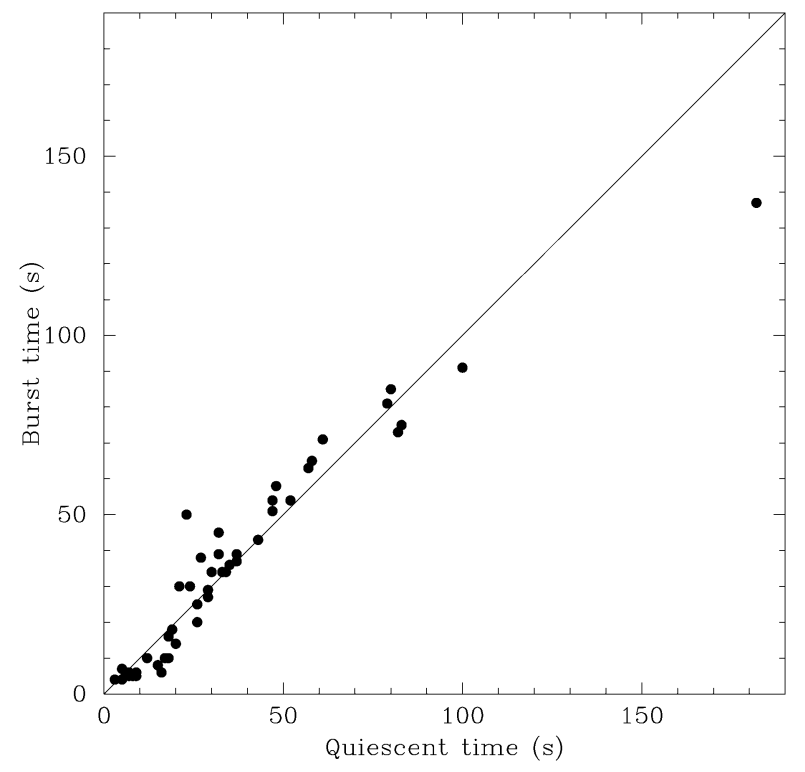

FIG. 2.-Correlation between burst and quiescent time (as defined in the text). The line is a $y=x$ relation.

radius of the accretion disk directly from the model without further assumptions.

The fitting procedure yielded spectral parameters for each of the 74 regions. By identifying the region of each $1 \mathrm{~s}$ bin, it is possible to produce a time history of relevant spectral parameters with a resolution of $1 \mathrm{~s}$. The inner radius and temperature are shown in the bottom two panels of Figure 1. As
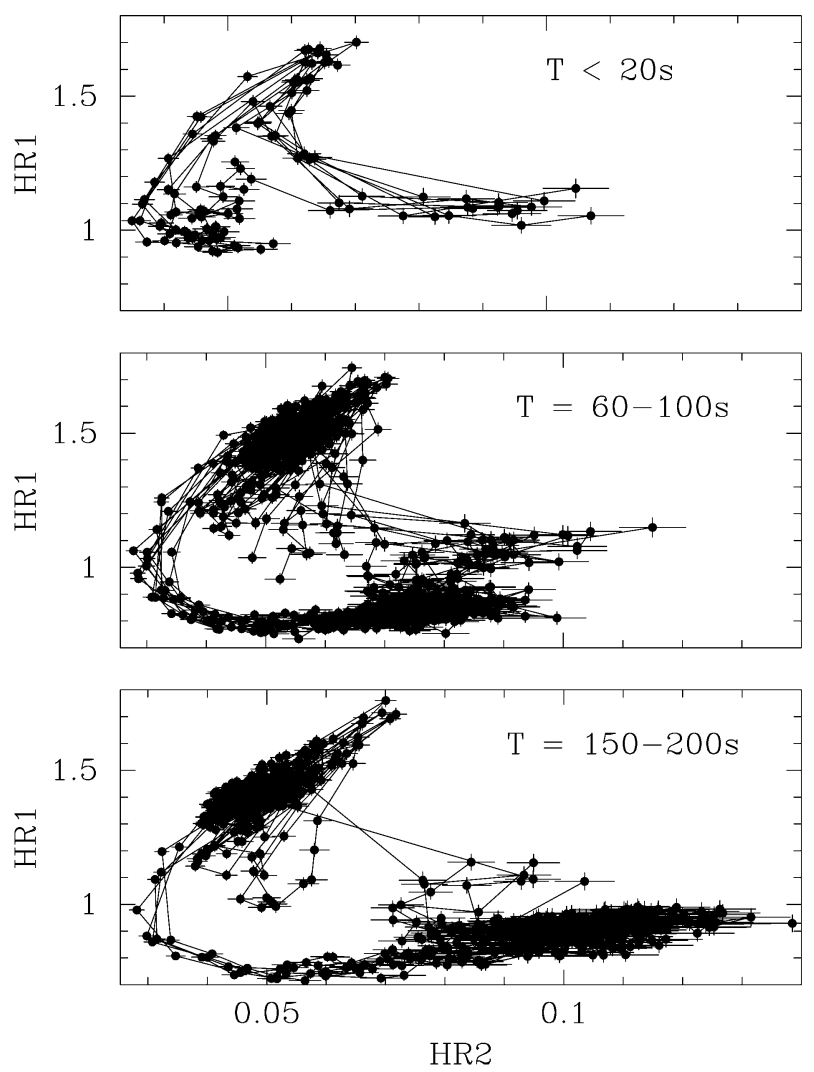

FIG. 3.-Three color-color diagrams for events grouped by total length 
can be seen, during bursts the temperature is above $2 \mathrm{keV}$ and the radius is stable around $20 \mathrm{~km}$, parameters consistent with those reported in Paper I. During quiescent phases, the temperature drops to lower values and the radius increases. There is a strong correlation between the length of the event and the largest radius reached (Fig. 4). In order to check the results of our "grid" procedure, we extracted spectra for the longest event in $16 \mathrm{~s}$ bins from the Standard 2 data, which have a much better energy resolution. We obtained similar results. Since the spectral parameters coming from these data are more accurate, we used those for the estimate of the values of the accretion rate.

Notice that the radii determined for the disk hole are smaller than that reported in Paper I, primarily because of the new PCA calibrations. Because of these differences, the behavior of the accretion rate derived from the model fits (which depends on the third power of the inner radius) is now radically different: the value of $\dot{M}$ during the quiescent phase is around 1.7-10 $\times 10^{-8} M_{\odot} \mathrm{yr}^{-1}$ (the range being determined by the spin of the central black hole, which determines the innermost stable orbit), while in the burst phase it is around a factor of 2 higher and variable.

We produced similar color-color diagrams for a number of other RXTE observations of GRS 1915+105. All observations we analyzed follow the pattern outlined above, except that of 1996 June 16 (Greiner et al. 1996). All the quasi-periodic bursts observed in many observations (see Taam et al. 1997) are consistent with repetitive short events like the ones described here.

\section{DISCUSSION}

The results described in the previous section can be interpreted within the model presented in Paper I, providing a unified picture of the variability observed in GRS 1915. In Paper I, we modeled the large amplitude changes as emptying and replenishing of the inner accretion disk caused by a viscousthermal instability. The small radius observed during the quiescent period was identified with the innermost stable orbit around the black hole, while the large radius during the burst phase was the radius of the emptied section of the disk. The smaller oscillations were interpreted as failed attempts to empty the inner disk. As can be seen from Figure 1, from this observation we find that all variations, from major events like the ones described in Paper I to small oscillations observed at the end of a large event, can be modeled in exactly the same fashion. In this scenario, the "flare state" presented in Paper I is simply a sequence of small events following a big one, similar to the small oscillations in Figure 1.

Both the spectral evolution and the duration of the event are determined by one parameter only, namely, the radius of the missing inner section of the accretion disk. For a large radius, the drop in flux will be large and the time needed for refill will be long. Following Paper I, it is natural to associate the length of the quiescent part of an event, $t_{q}$, to the viscous timescale of the radiation-pressure-dominated part of the accretion disk. This can be expressed as $t_{\text {visc }}=R^{2} / \nu$, where $\nu=\alpha c_{S} H$. From Frank, King, \& Raine (1992) the scale height $H$ and sound speed $c_{S}$ can be found, which leads to

$$
t_{\mathrm{visc}}=30 \alpha_{2}^{-1} M_{1}^{-1 / 2} R_{7}^{7 / 2} \dot{M}_{18}^{-2} \mathrm{~s},
$$

where $\alpha_{2}=\alpha / 0.01, R_{7}$ is the radius in units of $10^{7} \mathrm{~cm}, M_{1}$ is the central object mass in solar masses, and $\dot{M}_{18}$ is the accretion rate in units of $10^{18} \mathrm{~g} \mathrm{~s}^{-1}$. Notice that even the largest radii

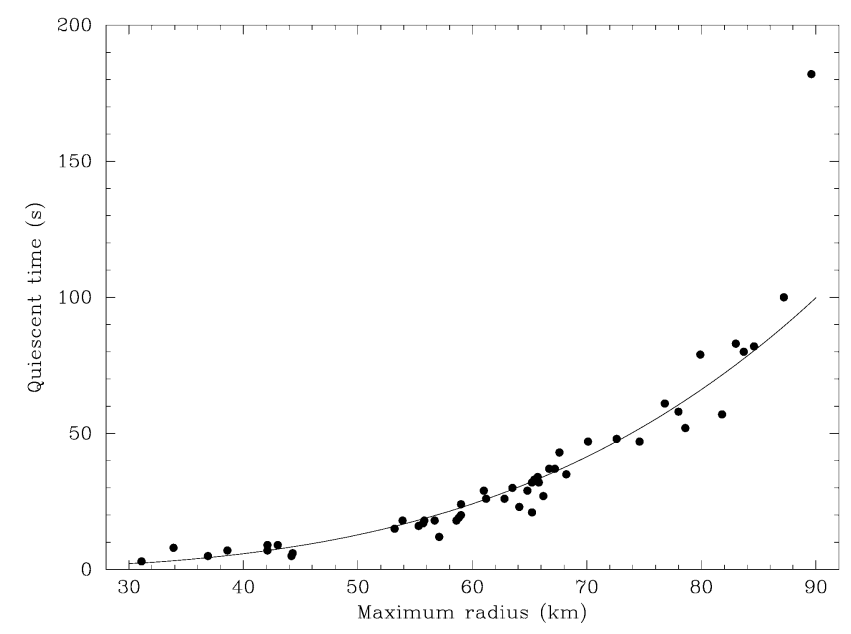

FIG. 4.-Correlation between total length of the event and maximum inner radius of the disk. The line is the best fit with a power law with fixed index $\gamma=3.5$ and normalization $1.44 \times 10^{-5}$. The last point has been excluded from the fit.

derived here are well within the radiation-pressure-dominated part of the disk (see eq. [2] in Paper I). The line in Figure 4 represents the best fit to the data with a relation of the form $t_{q} \propto R^{7 / 2}$. The fit is excellent, with the exception of the point corresponding to the longest event. The qualitative agreement with the theoretical expectation is striking, although by substituting the appropriate values for the mass and accretion rate, we find that our best fit predicts rather small values of $\alpha_{2}(0.004$ and 0.05 for the Schwarzschild and extreme Kerr cases, respectively). This indicates a small viscosity in the disk, although we stress that $t_{\text {visc }}$ is only a timescale, so additional corrections might be necessary in order to allow a precise quantitative comparison.

An event can therefore be pictured in the following way. At the start of a quiescent period, the disk has a central hole whose radius is $R_{\max }$. The hole is either empty or filled with gas whose radiation is too soft to be detected. Slowly, the disk is refilled by a steady accretion rate, $\dot{M}_{0}$, from outside. Each annulus of the disk will move along the lower branch of its $S$ curve in the $\dot{M}-\Sigma$ plane trying to stabilize at $\dot{M}_{0}$ (see Paper I). The surface gravity increases as the annulus moves toward the unstable point at a speed determined by the local viscous timescale. During this period, no changes are observed in the radius of the hole, since all the matter inside does not radiate in the PCA band. The observed accretion rate is $\dot{M}_{0}$. At some point, one of the annuli will reach the unstable point and switch to the high- $\dot{M}$ state, where the accretion rate is larger than $\dot{M}_{0}$, causing a chain reaction that will "switch on" the inner disk. The observed accretion rate is now higher than the external value, $\dot{M}_{0}$. A smaller, hot radius is now observed. At the end of the outburst, the inner disk runs out of fuel and switches off, either jumping back to the $\dot{M}<\dot{M}_{0}$ state or emptying completely. A new hole is formed, and a new cycle starts. Notice that in this scenario, the more "normal" state for the source is the one at high count rates, where the disk extends all the way to the innermost stable orbit: in this state the energy spectrum is similar to that of conventional black hole candidates (see Tanaka \& Lewin 1995).

Not only the start and end of a major burst but also all the small-amplitude oscillations within a burst show the same timing signature of decaying faster than they rise. This is in agree- 
ment with what was already noticed in Paper I: the rise time is determined by the speed at which a heating wave moves through the central disk, while the faster decay time is due to the rapid fall of matter into the black hole (or into a relativistic jet).

Chen et al. (1997) found that when HR2 exceeds 0.1, the power density spectrum is similar to the one observed in black hole transient systems during the very high state (see van der Klis 1995). In these occasions, a strong 1-6 Hz QPO peak was found, positively correlated with the count rate. Notice that the limit HR2 $>0.1$ is an indication that the source was in a quiescent state. Our spectral results show that the fast timing features (both QPO and band-limited noise; see Morgan et al. 1997) cannot originate from the innermost regions of the optically thick accretion disk, since those are missing during the quiescent phases. The fact that the QPO frequency increases with count rate is in qualitative agreement with the model, since a higher count rate indicates a smaller inner disk radius and, therefore, shorter timescales.

The radii for the disappearing region of the disk found here are considerably smaller than that reported in Paper I. This is entirely due to the improved knowledge of the spectral response of the PCA. During the burst phase, the accretion rate through the optically thick accretion disk is found to be higher than in the quiescent phase, contrary to what we reported in Paper I.

Although we can model the spectral evolution and the timescale of the events, most of the variability is yet to be explained. The main question to be answered is, what determines the length of the following outburst? Or, in more physical terms, what determines how large the next missing section of the disk will be? In some observations the events are very regular, in some others they are extremely random, and in some others no events are observed at all. In the observation reported here, a striking one-to-one relation between quiescent and burst time is observed, a relation that applies to the "outburst" and "quiescent" states of the observation presented in Paper I but is obviously not satisfied in other observations (see, e.g., Taam et al. 1997) nor during the "flare" state of Paper I. Moreover, as already mentioned, one observation among those we analyzed does not fit this pattern and requires a different interpretation (1996 June 16). Nevertheless, the model sketched here provides a satisfactory interpretation of the cause of the changes in the X-ray emission. It fits not only the energy spectral distribution but also the complex variability shown by GRS $1915+105$.

M. M. is a fellow of the Consejo Nacional de Investigaciones Científicas y Técnicas de la República Argentina. This work was supported in part by the Netherlands Organization for Scientific Research under grant PGS 78-277 and the Netherlands Foundation for Research in Astronomy under grant 781-76017. A. R. K. gratefully acknowledges a Particle Physics and Astronomy Research Council senior fellowship. J. v. P. acknowledges support from NASA under contract NAG 5-3003. We thank Keith Jahoda for helping with the calibration of the PCA data and the RXTE team for making these data publicly available. We thank D. Battacharya for his careful reading of the manuscript.

\section{REFERENCES}

Bailyn, C. D., Orosz, J. A., McClintock, J. E., \& Remillard, R. A. 1995, Nature, 378, 157

Belloni, T., Méndez, M., King, A. R., van der Klis, M., \& van Paradijs, J. 1997, ApJ, 479, L145

Castro-Tirado, A. J., Brandt, S., \& Lund, S. 1992, IAU Circ., 5590

Chen, X., Swank, J. H., \& Taam, R. E. 1997, ApJ, 477, L41

Frank, J., King, A., \& Raine, D. 1992, Accretion Power in Astrophysics (Cambridge: Cambridge Univ. Press)

Greiner, J., Morgan, E., \& Remillard, R. A. 1996, ApJ, 473, L107

Mirabel, I. F., \& Rodríguez, L. F. 1994, Nature, 371, 46
Mitsuda, K., et al. 1984, PASJ, 36, 741

Morgan, E., Remillard, R. A., \& Greiner, J. 1997, ApJ, 482, 993

Taam, R. E., Chen, X., \& Swank, J. H. 1997, ApJ, 485, L83

Tanaka, Y., \& Lewin, W. H. G. 1995, in X-Ray Binaries, ed. W. H. G. Lewin, J. van Paradijs, \& E. P. J. van den Heuvel (Cambridge: Cambridge Univ. Press), 2

van der Klis, M. 1995, in X-Ray Binaries, ed. W. H. G. Lewin, J. van Paradijs, \& E. P. J. van den Heuvel (Cambridge: Cambridge Univ. Press), 252

Zhang, S. N., Wilson, C. A., Harmon, B. A., Fishman, G. J., Wilson, R. B., Paciesas, W. S., Scott, M., \& Rubin, B. C. 1994, IAU Circ., 6046 